\title{
The New Worlds Observer: a mission for high-resolution spectroscopy of extra-solar terrestrial planets
}

\author{
Willard L. Simmons ${ }^{a}$, Webster C. Cash $^{b}$, Sara Seager ${ }^{c}$, Erik Wilkinson ${ }^{d}$, N. Jeremy Kasdin ${ }^{a}$, \\ Robert J. Vanderbei ${ }^{e}$, Naomi $\mathrm{Chow}^{a}$, Erica Gralla ${ }^{a}$, and Johanna Kleingeld ${ }^{a}$ \\ ${ }^{a}$ Mechanical and Aerospace Engineering, Princeton University, Princeton NJ 08544, USA; \\ ${ }^{b}$ Center for Astrophysics and Space Astronomy, University of Colorado, \\ Boulder CO 80309-0389, USA; \\ ${ }^{c}$ Carnegie Institution of Washington, Department of Terrestrial Magnetism, \\ 5241 Broad Branch Rd. NW Washington, D.C. 20015, USA; \\ ${ }^{d}$ Ball Aerospace, P.O. Box 1062, Boulder CO 80301; \\ ${ }^{e}$ Operations Research and Financial Engineering, Princeton University, \\ Princeton NJ 08544, USA
}

\begin{abstract}
The New Worlds Observer (NWO) is a proposed space mission to provide high resolution spectroscopy from the far UV to the near IR of extra-solar terrestrial sized planets. The design of NWO is based on the concept of a large, space-based, pinhole camera made up of two spacecraft flying in formation. The first spacecraft is a large, thin occulting shield (perhaps hundreds of meters in diameter) with a shaped "pinhole" aperture about $10 \mathrm{~m}$ in diameter. The second spacecraft is a conventional-quality space telescope (possibly with a $10 \mathrm{~m}$ primary mirror) which "flies" through the pinhole image of the planetary system to observe the extra-solar planets free from stellar background. In this paper we describe the design of the two spacecraft system. In particular, the shaped-pinhole design utilizes the shaped-pupil coronagraph pioneered for the Terrestrial Planet Finder. In this paper we describe some of the NWO's technology challenges and science opportunities. Additionally, we describe an extension of the design to provide $100 \mathrm{~km}$ resolution images of extra-solar planets.
\end{abstract}

Keywords: spectroscopy, space observatories, astronomy, extra-solar planets, exo-planets, formation flying spacecraft, shaped-pupil coronagraph, pinhole camera, planet finding

\section{INTRODUCTION}

Ever since the first detections of Jupiter-like extra-solar planets in 1995 new technologies and ideas have been proposed to detect and study different classes of planets. Currently under development is the Terrestrial Planet Finder (TPF) mission. The two current competing designs of TPF (a shaped-pupil coronagraph ${ }^{1,2}$ and a formation flying interferometer) will have the remarkable capability to detect small Earth-like ("terrestrial") planets orbiting nearby stars. However, they will have limited capability of characterizing those planets. In this paper we propose an idea for a follow-on mission to TPF which will have the ability to accurately map planetary systems, perform high-resolution spectroscopy of extra-solar planets, and possibly provide exo-planet images with $100 \mathrm{~km}$ resolution.

We have named our mission The New Worlds Observer (NWO). The basic concept of NWO is based one of the simplest imaging devices: the pinhole camera. In simple terms, a pinhole camera is created by puncturing a relatively small hole in an opaque screen and placing photographic film behind the hole at a particular focal

Further author information:

W.L.S.: E-mail: wsimmons@princeton.edu, Telephone: +1 (609) 258-7564

W.C.C.: E-mail: cash@casa.colorado.edu, Telephone +1 (303) 492-4056

N.J.K.: E-mail: jkasdin@princeton.edu, Telephone: +1 (609) 258-5673

R.J.V.: E-mail: rvdb@princeton.edu, Telephone: +1 (609) 258-0876

S.S.: E-mail: seager@dtm.ciw.edu, Telephone: +1 (202) 478-8868 


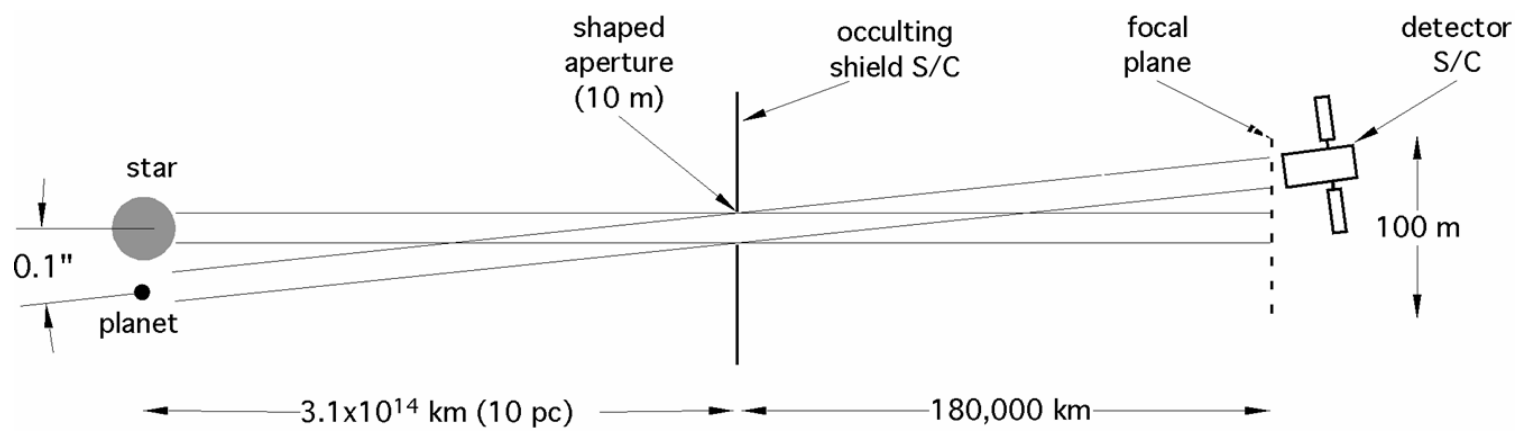

Figure 1. 1-D schematic of the design of the New Worlds Observer. The occulting shield spacecraft (the "starshade") is shown in the center of the diagram. A shaped aperture pupil acts like a lens, projecting an image 180,000 km away where it is observed by a detector spacecraft.

distance (related to the radius of the hole). Pinhole cameras have some very distinct advantages which we will take advantage of: they introduce no wavefront distortion, create no non-uniformity of transmission, and have almost no dust or cleanliness issues. In many ways, pinholes are the perfect lens. ${ }^{3,4}$

Taking this idea to an extreme, NWO is envisioned as a large space-based shaped-pinhole camera made up of a formation of two spacecraft (see figure 1). The first spacecraft is a large thin occulting shield, or "starshade". The starshade will have an approximately 10 meter "pinhole" in the center and be (perhaps) 100's of meters in diameter. Enhanced with optimal shaped-pupil coronagraphy techniques pioneered for TPF ${ }^{5}$ the pinhole will act like an ideal lens, projecting an "image" thousands of kilometers behind the starshade. The second spacecraft, a conventional-quality telescope, could then "fly" through the image of the alien planetary system to collect photons from the target planets. We anticipate that the imaging spacecraft would be able to collect enough photons to determine the composition, weather and rotation rate of each planet. This data would give us clues to whether or not life might exist on the planet. ${ }^{6-9}$

A key design requirement of both TPF and NWO is that must be separate the extremely bright starlight from the dim reflected light of a terrestrial planet. In the visible wavelengths, the required intensity contrast
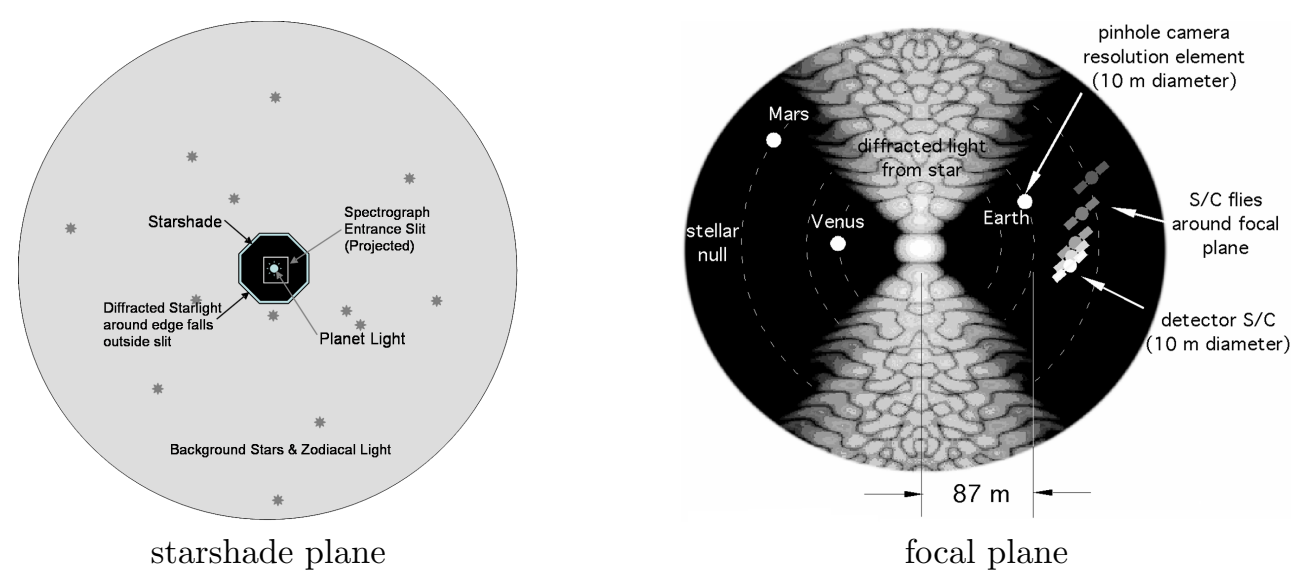

Figure 2. This diagram is a $2-\mathrm{D}$ slice of the starshade and focal planes shown in figure 1 . The image on the left shows what the starshade might look like as observed from a distant detector spacecraft. The image on the right is the focal plane image. In the focal plane, the detector spacecraft (denoted "S/C") "flies" through the image to observe the light diffracted from the planets. In this example a Spergel Pupil is used for the starshade resulting in the starlight being diffracted upwards and downwards. This diagram shows how our own planetary system might appear to an alien observer using a "New Worlds Observer-like" system. 
is $10^{-10} .^{10}$ In order to achieve this, it is necessary to make clever use of diffraction principles to prevent the starlight from "bleeding over" into the planet light. Several papers have shown this level of contrast is possible by using optimization mathematics to design a specially shaped entrance pupil. ${ }^{1,5,11,12}$ The NWO applies the same shaping techniques to provide high contrast with a large pinhole camera.

This paper contains a description of some of the technology challenges and expected science for The New Worlds Observer. In addition, we include a section devoted to extending the mission to perform Exo-Planet Imaging. We expect that by using a formation of two starshades, two collector craft, and one beam combiner craft, we will be able to image distant extra-solar planets with $100 \mathrm{~km}$ resolution. With a greater number of starshades and beam combiners we suspect that we could theoretically improve the resolution of the imaging system to as small as $1 \mathrm{~km}$ (although this would be extremely complex and expensive).

\section{TECHNOLOGY CHALLENGES}

There are many technical challenges in the development of NWO. Only three major ones will be addressed here: The design of the starshade, the design of the spectrometry/detector spacecraft, and preliminary formation flying and orbit design.

\subsection{Starshade Design}

The starshade (refer to Figures 1 and 2) is envisioned as a large (100's of meters in diameter), lightweight, thin, and opaque screen. The starshade must also be dark to prevent unwanted reflection of star and sun light into the focal plane. The key feature of the starshade is that it contains a specially shaped pinhole. This pinhole must be optimally designed to provide a visible light contrast level of $10^{-10}$ in the focal (observation) plane.

The design of pinhole shapes leverages off previous studies of optimal shaped-pupil coronagraphs for the Terrestrial Planet Finder (TPF). Kasdin, Vanderbei and Spergel demonstrated that it is possible to provide the necessary $10^{-10}$ contrast level by using specially shaped pupil masks. ${ }^{1,2,5,12}$ Some simple examples of the pupil designs are shown in Figures 3 and 4. As this paper is being written laboratory experiments are taking place to verify the contrast produced by the shaped pupil coronagraph designs. Results will be available in future publications.

Using these shaped pupils as a starting point, the optimal overall starshade design for NWO is currently being investigated. For a first cut, the starshade was assumed to be of infinite-extent with a shaped-pinhole in the center. Trading-off integration times, focal distance and angular resolution, we arrived at a baseline pinholepupil size of $10 \mathrm{~m}$ and several possible optimized shapes. As expected, the shapes are qualitatively similar to the shapes discovered in the TPF coronagraph mask studies. A simplified procedure for setting up this type of optimization problem is presented below. More details are available in Vanderbei (2003) and (2004). ${ }^{5,11,12}$

The pupil shapes are developed in a two-stage optimization problem. First, we assume that the pinhole is not actually shaped, but smoothly apodized (continuous functions are almost always easier to optimize). In
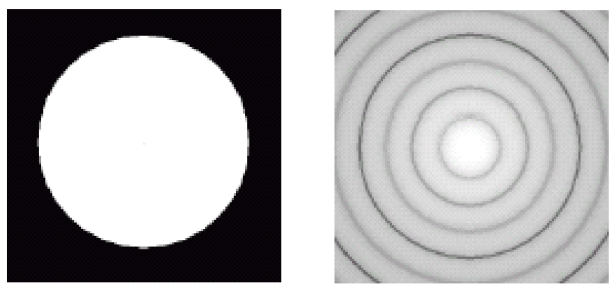

Round Pupil

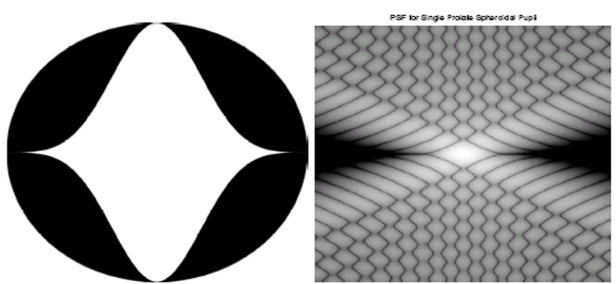

Spergel-Kasdin Pupil

Figure 3. The images above show two examples of pupil shapes and their associated log-scaled point spread functions (PSF). The dark regions in the PSF indicate the area where the contrast level is better than $10^{-10}$. From inspection, the round pupil obviously does not provide adequate contrast to detect terrestrial planets. However, note that the Spergel-Kasdin pupil does have suffiently dark area, but only inside two symmetric angular regions. ${ }^{1}$ 


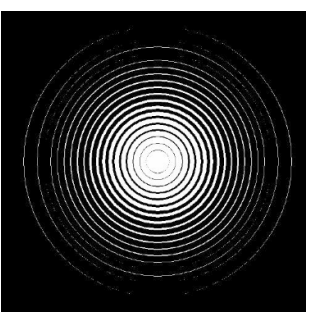

Concentric Rings Pupil

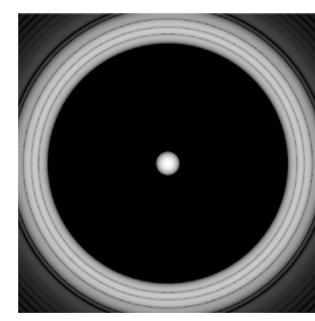

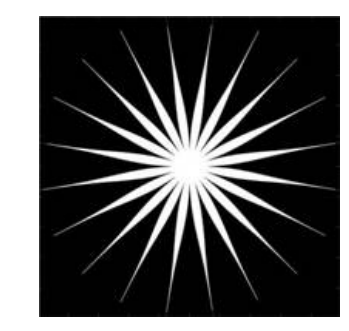

Star-Shaped Pupil

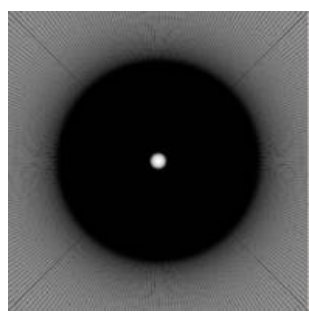

Figure 4. Examples of two different circularly-symmetric shaped pupils. Both of these pupil designs have a 360 degree region of high contrast and a tighter inner working distance than the Spergel pupil. The main disadvantage of these pupil design is that they have a lower total throughput (this means longer integration times). The star-shape pupil shown on the left is a 20-point design shown for clarity. The accompanying PSF shown on the far right is for a 150-point star. This is the minimum number of points needed to meet the contrast requirements in the entire dark region. ${ }^{5}$

formulating the problem we assume the apodization affects only the amplitude of the incoming wave and does not affect its phase. This turns out to be a legitimate assumption because of the way the apodization function is eventually used.

In a second run of the optimization we then map the magnitudes of the apodization function to a discontinuous shape. We use the apodization as a starting point and a formula relating its magnitude to a pattern such as a star-shape or a concentric ring pattern (detailed in ${ }^{5,11}$ ). The following is a simplified summary of how the first stage of the optimization problem is formulated. The equation for the electric field projected into the focal plane is:

$$
E(\xi, \eta)=\int_{-a}^{a} \int_{-b}^{b} e^{i k(d((x, y),(\xi, \eta))-f)} A(x, y) d x d y
$$

where:

$$
\begin{array}{ll}
E(\xi, \eta) & \text { is electric field in the focal plane } \\
k=2 \pi / \lambda & \text { is the wave number } \\
d(\cdot, \cdot) & \text { is the distance from the screen to the focal plane } \\
A(x, y) & \text { is the apodization function }(0<A(x)<1)
\end{array}
$$

Through a series of steps and using the assumption of radial symmetry, these integrals can be simplified to become parametric single integrals of one variable:

$$
E(\rho)=\int_{0}^{D / 2} \exp \left(\frac{\imath k r^{2}}{2 f}\right) J_{0}\left(\frac{k r \rho}{f}\right) A(r) r d r,
$$

where $D$ is the diameter of the aperture, $r$ is the radial position in the pupil plane and $\rho$ is the radial position in the focal plane. The diffraction equation is now a single integral and a parametric function of a single variable.

An optimization problem requires a cost function; for this problem we usually choose to maximize the throughput of the pinhole defined by a function such as:

$$
\text { throughput }=\int_{D / 2}^{-D / 2} A(r) 2 \pi r d r
$$

The most important constraint used in this optimization is to require the contrast in the point spread function to be high enough to distinguish the planets from the parent star. This is held within a particular range of inner working distance (iwd) and outer working distance (owd):

$$
|E(\xi)| \leq 10^{-5} E(0), \quad \text { when iwd } \leq \xi \leq \text { owd }
$$


Formulating these problems in a computationally stable and tractable way has proven to be a non-trivial task. This problem is highly non-linear and non-convex, so it must be broken up into separate solution stages and formulated in a careful way.

The next (and more difficult) step is to discover the dimensions and optimum shape for the "fringe" of the starshade. This is a more difficult problem, as it requires the use of near-field (Fresnel) diffraction theory rather than only far-field (Fraunhoffer) theory. Without shaping the fringe the starshade must be 100 times the diameter of the pinhole. Using a $10 \mathrm{~m}$ pinhole, this would mean we would need a giant screen $1 \mathrm{~km}$ in diameter. Although it may be possible to construct a starshade of this size in space we believe that using shaped fringes to optimize the diffraction we could reduce the diameter down to a much more manageable size: perhaps $5 \mathrm{x}$ to 10x the diameter (50m to $100 \mathrm{~m})$.

As the size and shape of the starshade becomes more clearly defined, the mechanical design can progress. It is important for the starshade to satisfy its optical requirements, but it must also be small and light enough so that it can be launched into space for a reasonable cost. We envision that the starshade will have to fold up and fit into the payload shroud of a large launch vehicle such as an Atlas V or Delta IV rocket. Assembly of separate pieces of the starshade on-orbit is a possible option, but will be avoided because of the high cost and complexity involved.

\subsection{Telescope \& Spectrometer Spacecraft}

The size of the pinhole aperture defines an effective resolution element at the collector spacecraft. Thus, the collector must have a telescope aperture comparable to the size of the pinhole aperture to maximize sensitivity, i.e. collect all the light within the resolution element. The role of the telescope is to collect the light from the pinhole aperture and feed the light into a photometer, spectrograph or relay optic, so the telescope need only image a very small field of view and can operate on-axis. This allows the use of simple two-element telescopes, e.g. a Cassegrain.

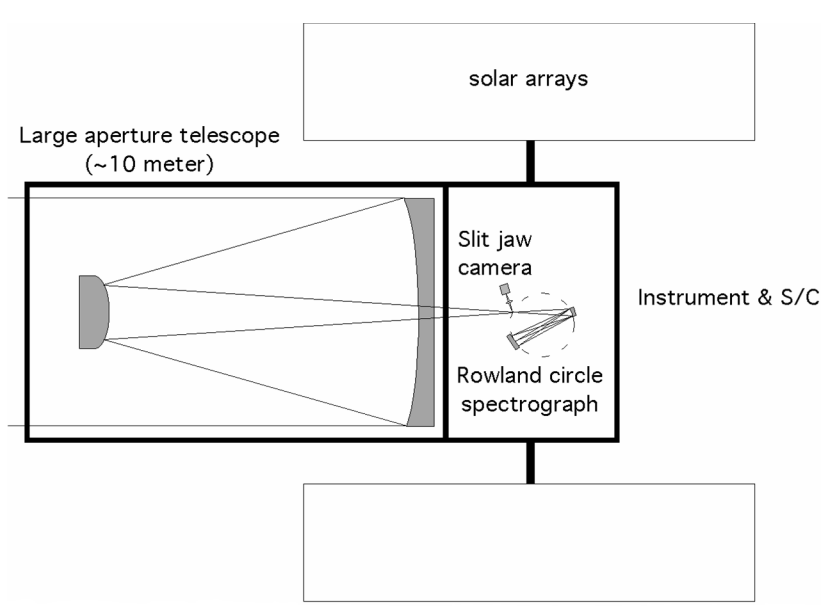

Figure 5. Schematic of the detector spacecraft. It is a conventional quality telescope with a Rowland circle spectrograph.
The size of the occulting shield surrounding the pinhole aperture defines the pointing requirement on the detector spacecraft and the size of the entrance aperture into the spectrograph or relay optic. For example, if the occulting shield is $1 \mathrm{~km}$ in diameter and the spacecraft is located $185,000 \mathrm{~km}$ away then the detector spacecraft must point to approximately 0.5 arcsec to insure that light from beyond the occulting shield does not enter the system, thereby degrading the planetary signal. This also demonstrates that the telescope need only support a small field of view. There is obviously a trade-off between the size of the occulting shield and spacecraft pointing control that will be studied. The detector used in the spectrograph must have an extremely low intrinsic background to minimize the noise in the spectrum, as the fluxes from the planet will be low.

With the basic requirements it is straightforward to estimate the required exposure times versus aperture diameter for the spectrograph. As a straw-man instrument we have assumed an instrument with a two-element telescope, and one of three moderately sized (approx. $100 \mathrm{~mm}$ with $0.010 \mathrm{~mm}$ pixels) detectors; glass microchannel plates, silicon microchannel plates, or a CCD. In addition, we are baselining a spectral resolution of 1000 across the band. We also assume a reflectivity of $90 \%$ for each reflection, a groove efficiency of $75 \%$, a detector quantum efficiency of $80 \%$, and an obscuration factor of $80 \%$. These values represent either currently demonstrated performance or assume a modest increase in performance expected as technology improves. The bandpass of the instrument will cover from $1150 \AA$ to $20,000 \AA$, thus including potential auroral emissions from $1216 \AA$ and the major biomarkers between $5000 \AA$ and $9000 \AA$. With these assumptions the net efficiency of the instrument is 
$\sim 0.35$. In reality different detectors may be required to cover the entire band-pass and there can be significant wavelength dependence in optics performance and detector quantum efficiencies, however, more detail will be forthcoming in the study.

The number of detected counts can be computed versus telescope collecting area along with intrinsic noise from the detector. Assuming the flux distribution shown in Figure 9 , and a spectral resolution element 0.03 $\mathrm{mm} \times 1 \mathrm{~mm}$ we calculate the telescope diameters and count rates (see Table 1).

Detector technology will play a crucial role in the instrument; intrinsic detector background can overwhelm the weak signals and force unacceptably long integration times (see Table 1). However, in the UV silicon MCPs are proving to be far superior to glass MCP detectors in their intrinsic background, due to the lack of radioisotope contamination in the silicon. Current silicon MCPs are demonstrating background rates of 0.02 counts $/ \mathrm{sec} / \mathrm{cm}^{2}$ compared to 0.5

Table 1. Summary of trade-off study of pinhole size, integration times, and detector types.

\begin{tabular}{||c|c|c|c|c||}
\hline $\begin{array}{c}\text { dia. } \\
(\mathrm{m})\end{array}$ & $\begin{array}{c}\text { req. integration } \\
\text { time (days) for } \\
100,000 \text { counts }\end{array}$ & $\begin{array}{c}\mathrm{MCP}_{\text {glass }} \\
\text { det. noise }\end{array}$ & $\begin{array}{c}\mathrm{MCP}_{\text {Si }} \\
\text { det. noise }\end{array}$ & $\begin{array}{c}\mathrm{CCD} \\
\text { det. noise }\end{array}$ \\
\hline 1 & 28 & 363 & 15 & 604800 \\
\hline 5 & 1.1 & 15 & 0.6 & 24192 \\
\hline 10 & .3 & 4 & 0.1 & 6070 \\
\hline 15 & .1 & 2 & 0.06 & 2700 \\
\hline 20 & .07 & 1 & 0.04 & 1512 \\
\hline
\end{tabular}

Assumptions: $\mathrm{F} \lambda \sim 3 \mathrm{e}-9$ photons $/ \mathrm{cm}^{2} / \mathrm{sec} / \AA ̊$ for Earth at $10 \mathrm{pc}, \delta \lambda=5 \AA$, system efficiency $\sim 0.35, \lambda=5000 \AA$. Background calculations done using MCPglass $=0.5$ counts $/ \mathrm{cm}^{2} / \mathrm{sec}, \mathrm{MCPsilicon}=0.02$ counts $/ \mathrm{cm} 2 / \mathrm{sec}$, $\mathrm{CCD}=1 \mathrm{e}-$ read noise and readout every 20 minutes, and a $0.03 \times 1 \mathrm{~mm}$ resolution element with $0.010 \mathrm{~mm}$ pixels. counts $/ \mathrm{sec} / \mathrm{cm}^{2}$ for glass MCPs. CCD detectors are unlikely to be suitable, but CMOS detector technology is advancing rapidly and remains very promising. In any event, we will thoroughly parameterize the required detector performance characteristics and evaluate current detector technology for suitability.

\subsection{Orbit Design}

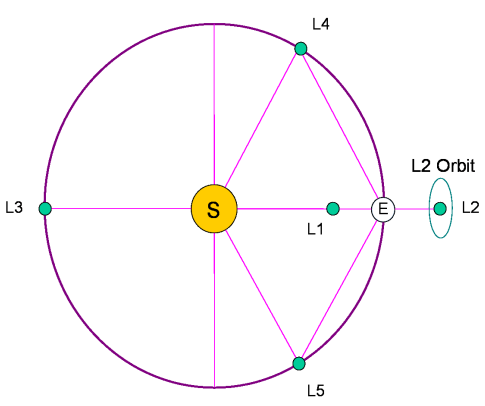

Figure 6. Lagrange points for Sun-Earth-Spacecraft System.
In this section, we examine potential orbits for the NWO. Orbit design issues are complicated by the necessity for formation flight of the detector and screen. However, the complexities of orbit design for this mission are simplified if we take advantage of the unique properties associated with Lagrange points. In a three-body system in which the two massive bodies have approximately coplanar and circular orbits (such as a Sun-Earth-Spacecraft system), five points exist where the gravitational forces acting on the third body balance the centripetal force required for rotation. Spacecraft placed at these Lagrange points thus remain in a fixed orientation with respect to both of the massive bodies. In the Sun-Earth-Spacecraft system, for example, a spacecraft placed at any of the Lagrange points orbits the Sun at the same rate as the Earth (see Figure 6). This is particularly advantageous for a mission such as the NWO because it allows for the telescope and camera to maintain a constant proximity to Earth, simplifying both launch and communication with the two spacecraft.

Rather than sitting directly at the Lagrange points, spacecraft enter periodic or semi-periodic 'orbits' about the point. Most previous Lagrange point missions utilized a special type called a halo orbit, which looks like an orbit about the edge of a potato chip. Halo orbits approximate the conventional planar orbit shape by minimizing the out-of-plane motion. Lissajous orbits, on the other hand, are three-dimensional. For this mission, a three-dimensional orbit is required, such that the NWO can have maximum coverage of space. We propose a Lissajous orbit about the L2 point (1.5 million $\mathrm{km}$ from Earth) because it offers good sky coverage. Placing a telescope at L2 will minimize interference from sunlight and eliminate direct obstruction from both the Earth and the sun. 
In this analysis a distance between the detector and screen is assumed to be fixed near $180,000 \mathrm{~km}$. The nominal time to collect spectroscopic data of a planet is assumed to be 0.3 days. (see Table 1 for a $10 \mathrm{~m}$ pinhole). Using these assumptions, an orbit has been designed where the detector craft (located at L2) would have to move at a rate of approximately $20 \mathrm{~m} / \mathrm{s}$ to maintain a constant relative position to the detector screen. This speed can easily be achieved and maintained with electric propulsion thrusters. The orbit is depicted in Figure 7. Note that this preliminary orbital analysis was done using linear approximations, and will be revised with all possible perturbations added.

Also notable in Figure 7 is that the current orbit design only allows for a particular "visibility angle". In further study we hope to use the natural precession of the orbit to rotate the Lissajous orbit to achieve complete sky coverage. Additionally, future study of orbit possibilities about the L4 and L5 points may provide better orbit design.

This early orbit study did not take into account the effect of solar pressure on the starshade. We do expect to be able to take advantage of this property to decrease the launch costs and reduce the propulsion needed for station keeping.

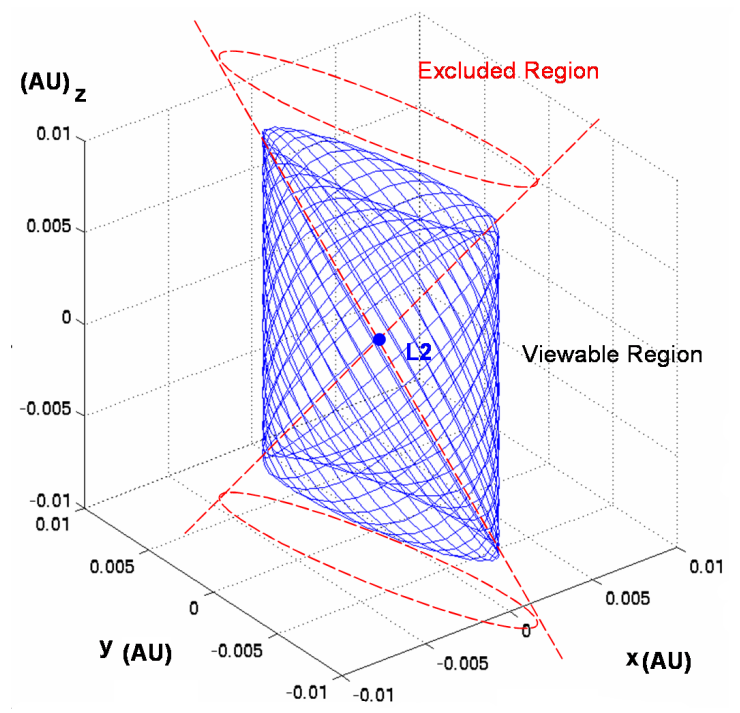

Figure 7. A preliminary proposed Lissajous orbit about the L2 Sun-Earth Lagrange point. The starshade traces out a cylindrical shaped path, while the dectector spacecraft remains close to the L2 point.

\section{SCIENCE OPPORTUNITIES}

The New Worlds Observer has the ability to provide unprecedented science data for extra-solar planets. In this section we outline three major functions of the mission: Mapping of planetary systems, high-resolution spectroscopy, and planet imaging.

\subsection{Planetary System Mapping}

Even if the Terrestrial Planet Finder has already observed a planetary system, it is likely that we will want to re-survey each planetary system using NWO's superior resolution. New Worlds can accumulate 15c/s from the Earth at 10pc in a photometric mode, so a strong detection takes only 7 seconds. A typical habitable zone spans about 5 square AU. Since the detector craft aperture subtends about $0.01 \mathrm{AU}^{2}$, it will take about one hour to survey. If the observation is done more slowly, the NWO would be sensitive to planets as small as our Moon. Once the survey is complete, New Worlds can return to the position of the most interesting planets, where it could spend a week or more collecting spectra and images, as dictated by the mission scheduling.

Figure 8 is a simulation of planetary system mapping. A code was used to simulate the data stream from an observation or our solar system at a distance of 10pc. It is clear that all the planets would be detectable with the likely exception of Mercury which is simply too close to the Sun.

\subsection{Exo-Planet Spectroscopy}

The New Worlds Observer can provide high resolution spectroscopy from the far UV to the near IR with excellent sensitivity. New Worlds will be able to detect methane, water, oxygen, ozone, and other gases at Earth's current and past levels of concentration. NW will have high enough sensitivity and spectral resolution to detect important atmospheric signatures and chlorophyll-type absorption edges. By operating at visible wavelengths (which penetrate to the ground) NW will be able to determine the planet's rotation rate, presence of weather, and even the existence of liquid oceans. Combined with atmospheric diagnostics, this information will extend the reach of biologists, geophysicists, and atmospheric chemists to worlds and ecosystems far beyond Earth. 

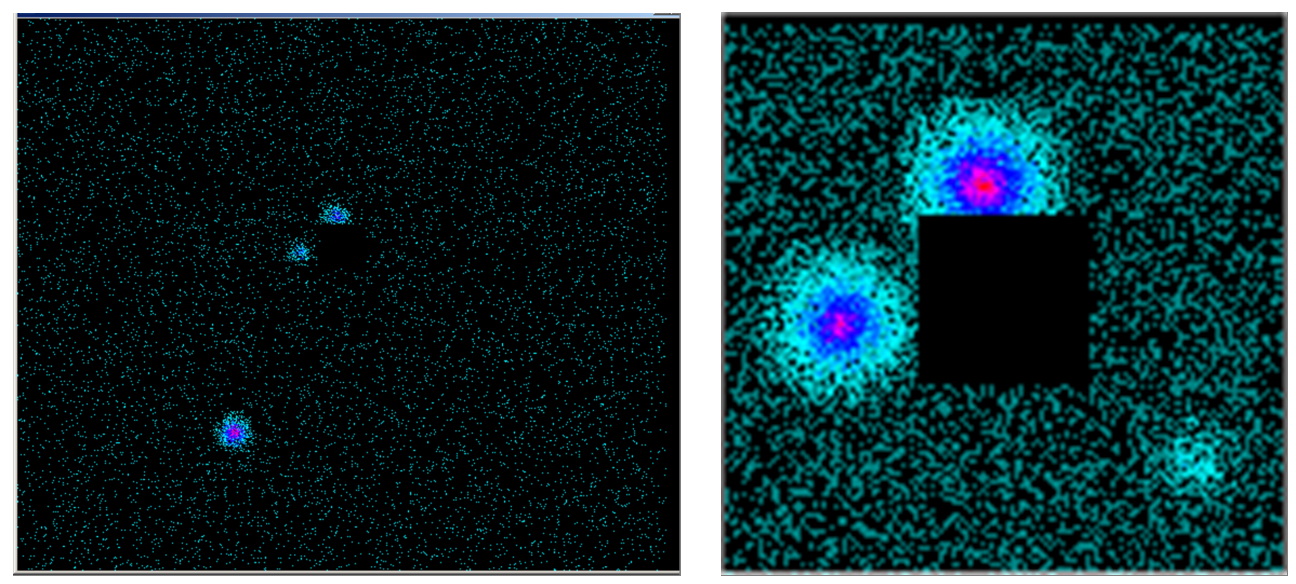

Figure 8. Simulations of planet system mapping with New Worlds. To the left a day is spent surveying the solar system out to $6 \mathrm{AU}$ as viewed from 10pc. Jupiter, Earth and Venus are visible. To the right, a day is spent surveying just the habitable zone. Venus, Earth and Mars are detected.

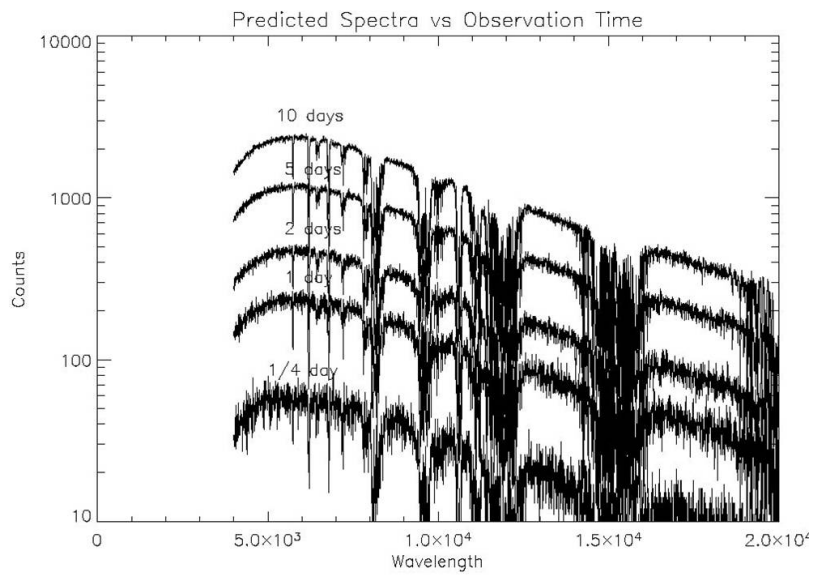

Figure 9. Simulated spectra of an Earth-like planet at 10pc taken with New Worlds.

Most studies of Earth-like planet biosignatures to date have focused on present-day Earth or small variations of it. But experience in planetary exploration and exo-planet detection amply shows we should not be limited to our only example of planetary life. Indeed Earth has, in the past, exhibited different atmospheric signatures due to extreme climatic states such as glaciation, the rise of photosynthetic organisms, and methane bursts. Moreover, just as each of our rocky planets differs greatly from each other, there is no reason to expect extrasolar terrestrial planets to be similar to Earth. If a planetary atmosphere is determined to have a severe departure from

Table 2. Spectroscopic Biomarkers.

\begin{tabular}{|ll|}
\hline Water & Necessary for habitability \\
Oxygen & Free oxygen results only from active plant life \\
Ozone & Results from free oxygen \\
Nitrous Oxide & Another gas produced by living organisms \\
Methane & Potential life indicator if free oxygen also present \\
Vegetation & Red edge of Earth's vegetation at 750nm \\
\hline
\end{tabular}




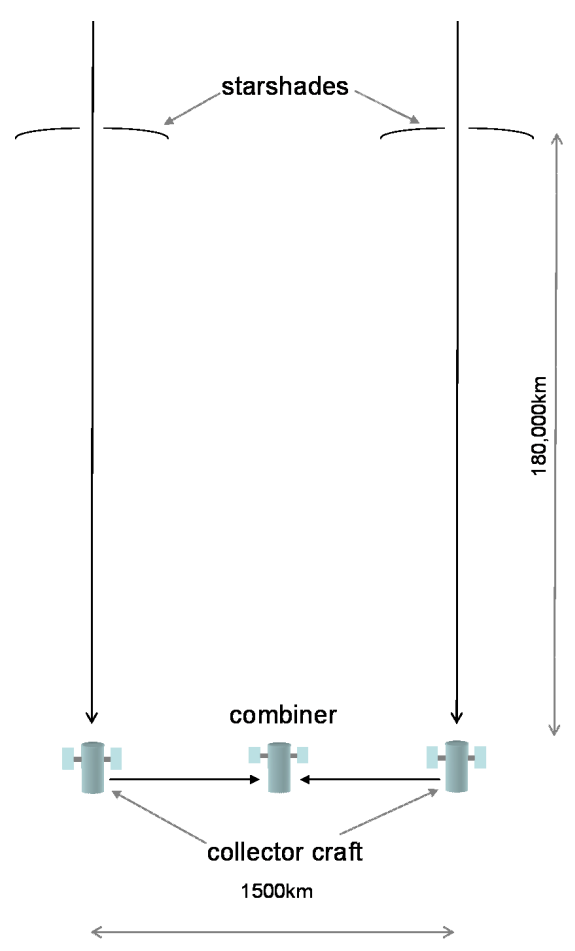

Figure 10. Schematic of the full imaging mode features two collector craft separated by $1500 \mathrm{~km}$, each shadowed by a starshade. The two beams are mixed in a fifth craft.

chemical and thermodynamic equilibrium would we be able to identify the disequilibrium features with biological modification of atmospheric composition? Or will there always be an ambiguity with geological processes? Will we be able to unambiguously identify a spectral signature not consistent with any known atomic, molecular, or mineral signature in the solar system and Universe (such as Earth's vegetation red edge)?

For a suitably bright planet, a time series of sufficiently high quality spectro-photometric data could reveal a wealth of physical characteristics at wavelengths that penetrate to the planetary surface. Visible wavelengths are more suited for these measurements than mid-IR wavelengths because the albedo contrast of surface components is much greater than the temperature variation across the planet's surface. Moreover, the narrow transparent spectral window at 8-12 microns will close for warmer planets than Earth and for planets with more water vapor than Earth.

\subsection{Exo-Planet Imaging}

With two craft (a shade and collector) we have New Worlds Observer, capable of mapping planetary systems and following up with spectroscopy and photometry. With five craft we can create the New Worlds Imager (NWI), designed to take high-quality pictures (100km resolution) of exo-planets. Ultimately resolution as fine as $1 \mathrm{~km}$ might be possible although the size requirement on such a system is daunting.

The overall architecture of the New Worlds Imager is presented in Figure 10. It involves five spacecraft flying in formation. Two starshades, two collector craft and one beam combiner craft. The starshade and collector craft work just as for the observer mode, except that the beam is not detected in the collector. Figure 11 shows that the beam from the planet must pass through the craft where it is picked off and recollimated into a beam that is directed to the combiner craft. The entire array is monitored with a laser metrology net and the craft are held in position using microthrusters. To maintain formation across such large distances it is likely that the telescope will have to be sent into a low gravity gradient sun-centered orbit.

In the combiner craft two telescopes gather the light from the collector craft as shown in Figure 11. The two beams are then mixed to form fringes on a detector. The fringe visibility is measured at many points in the UV 


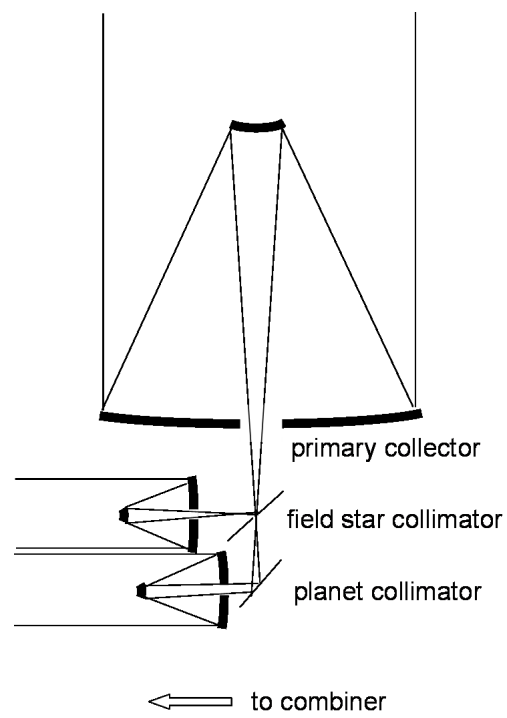

Figure 11. Schematic of the optics in the collector. Light from the planet and from a field star are re-directed to the beam combiner.

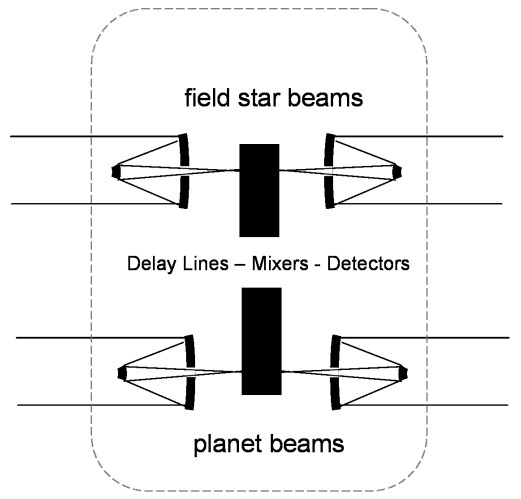

Figure 12. At the beam combiner more telescopes are needed to focus the wavefronts from the collector.

plane and then used to reconstruct an image in the manner of the radio astronomers.

For true planet imaging the goal would be to achieve $100 \mathrm{~km}$ resolution in the visible at a distance of $10 \mathrm{pc}$. That means a resolution of $3 \times 10^{-13}$ radians, implying that the baseline of the interferometer must be about $3 \times 10^{12} \lambda$ or $1500 \mathrm{~km}$ for yellow light. The diameter of a terrestrial planet is about 100 times that, so in order to fill in the UV plane, the interferometer also needs to operate at a distance as low as $15 \mathrm{~km}$. The beam combiner is on a separate craft half way between the two collectors in order that the total path from source to interferometer through the two apertures may be set equal.

The signal from the planet is passed through a collimator before it is sent to the beam combiner. To keep the beam from spreading significantly across the $1500 \mathrm{~km}$, the collimator must be a diffraction limited one meter aperture or larger - roughly 0.1 arcsecond quality.

At the combiner craft another telescope captures the (diffraction limited) wavefront from the collector craft and concentrates it. The two beams are mixed to create fringes. The fringe visibility is measured as a function of position in the UV plane and as a function of wavelength. The fringe visibilities are added in a manner similar to that performed in the radio to create the final image in a computer.

Figure 11 and 12 also show optics for a "field star". This is necessary for maintenance of pointing. Consider that $100 \mathrm{~km}$ at $10 \mathrm{pc}$ represents 0.1 micro-arcseconds resolution, so the slightest drift in pointing can ruin the 
image. The idea for maintenance of pointing is to use a field star, one that is outside the area occulted by the starshade but close enough to the center of the field of view of the collector scope that it remains diffraction (as opposed to aberration) limited. The light from such a (presumably distant) star is picked off at the focal plane and sent through a separate collimator to the beam combiner craft. In the combiner craft there is a separate pair of telescopes that collect the star light and create fringes.

The problem is not so much the drift in the distance from the combiner to the collectors, as that can be directly measured in the metrology net. The problem is the distance from the source (planet) to the collector. If the collector drifts toward the source or away, by even a micron, the fringes will shift in the combiner. The star, which is typically 20th magnitude is thousands of times brighter than the planet, and as a result will be able to rapidly detect a fraction of a wavelength shift in the total optical path difference (OPD).

In practice it will be necessary to study the time scales and possible approaches to keeping the OPD small. For example, the beam combiner could contain a delay line similar to that in use on SIM (Space Interferometry Mission) that can compensate for smaller motions. On the other hand, small adjustments might be easier to make with micro-thrusters on the collector craft. Finding the zero OPD will be challenging. A number of approaches will be studied.

\section{FINAL REMARKS}

The New Worlds Observer is a rapidly progressing design for an exo-planet science mission. It not only presents the exciting possibility of mapping planetary systems, but it could also provide the first ever images of an extra-solar planet's oceans and continents. Even if the imaging configuration is not built, the NWO will provide spectroscopic data which will give us unprecedented information about the chemical makeup of planets outside of our solar system.

A similar concept to the NWO is the Big Occulting Steerable Satellite (BOSS) proposed by Copi and Starkman. ${ }^{13}$ This project is also a large screen flown in space in front of a telescope. The main difference is that their design is an occulter, and not a pinhole camera. It can be thought of as the "opposite" or "dual" of the NWO design. We are currently working to compare and contrast both ideas to determine if an occulter or a pinhole camera is the best solution for extra-solar planet studies.

\section{REFERENCES}

1. N. Kasdin, R. Vanderbei, D. Spergel, and M. Littman, "Extrasolar planet finding via optimal apodized-pupil and shaped-pupil coronagraphs," Astrophysical Journal 582, pp. 1147-1161, January 2003.

2. M. Kuchner and D. Spergel, "Terrestrial planet finding with a visible light coronagraph," in Scientific Frontiers in Research on Extrasolar Planets, ASP Conference Series 294, 2003.

3. M. Young, "Pinhole optics," Applied Optics 10, pp. 2763-2767, December 1971.

4. R. D. Guenther, Modern Optics, Wiley, 1990.

5. R. J. Vanderbei, D. N. Spergel, and N. J. Kasdin, "Circularly symmetric apodization via starshaped masks," Astrophysical Journal 599, pp. 686-694, December 2003.

6. D. J. D. Marais, M. Harwit, K. Jucks, J. Kasting, D. Lin, J. Lunine, J. Schneider, S. Seager, W. Traub, and N. Woolf, "Remote sensing of planetary properties and biosignatures on extrasolar terrestrial planets," Astrobiology 2, 153, 2002.

7. N. Woolf, P. Smith, W. Traub, and K. Jucks, "The spectrum of earthshine: A pale blue dot observed from the ground," Astrophysical Journal 574, pp. 430-433, July 2002.

8. L. Arnold, S. Gillet, O. Lardire, P. Riaud, and J. Schneider, "A test for the search for life on extrasolar planets: Looking for the terrestrial vegetation signature in the earthshine spectrum," Astronomy $\& 3$ Astrophysics 392, p. 231, 2002.

9. E. Ford, S. Seager, and E. Turner, "Characterization of extrasolar terrestrial planets from diurnal photometric variability," Nature 412, pp. 885-887, 2001.

10. C. A. Beichman, N. J. Woolf, and C. A. Lindensmith, The Terrestrial Planet Finder, JPL Publication 99-3, JPL, Pasadena, 1999. 
11. R. J. Vanderbei, D. N. Spergel, and N. J. Kasdin, "Spiderweb masks for high-contrast imaging," Astrophysical Journal 590, pp. 593-603, June 2003.

12. R. J. Vanderbei, N. J. Kasdin, and D. N. Spergel, "Rectangular-mask coronagraphs for high-contrast imaging," astro-ph/0401644, January 2004.

13. C. J. Copi and G. D. Starkman, "The big occulting steerable satellite (boss)," Astrophysical Journal 532, pp. 581-592, March 2000. 